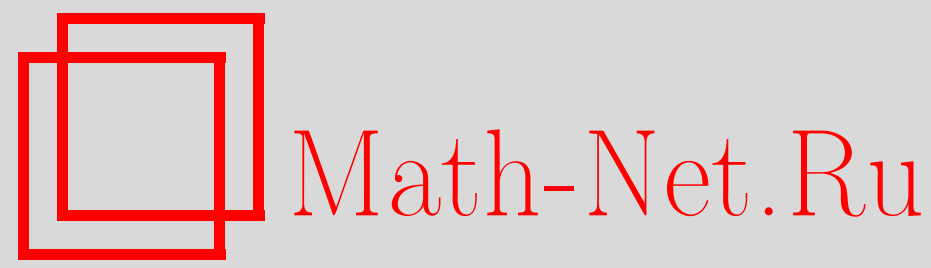

Г. Н. Аржанцева, А. Ю. Ольшанский, Общность класса групп, в которых подгруппы с меньшим числом порождающих свободны, Матем. заметкu, 1996, том 59, выпуск 4, 489-496

DOI: https://doi.org/10.4213/mzm1744

Использование Общероссийского математического портала MathNet.Ru подразумевает, что вы прочитали и согласны с пользовательским соглашением

http://www. mathnet.ru/rus/agreement

Параметры загрузки:

IP : 54.205 .225 .156

26 апреля 2023 г., 13:27:26

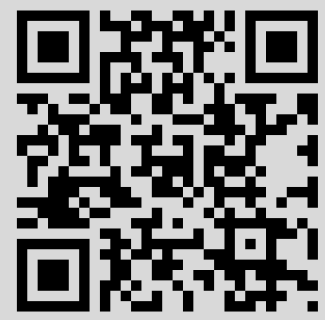




\title{
ОБШНОСТЬ КЛАССА ГРУПП, В КОТОРЫХ ПОДГРУППЫ С МЕНЬШИМ ЧИСЛОМ ПОРОЖДАЮЩИХ СВОБОДНЫ
}

\author{
Г.Н. Аржанцева, А. Ю. Ольшанский
}

0. Введение. Хорошо известно, что в фундаментальной группе ориентируемой замкнутой компактной поверхности рода $g$ все подгрупшы бесконечного индекса свободны. Отсюда следует, в частности, что в группе

$$
S_{g}=\left\langle a_{1}, \ldots, a_{g}, b_{1}, \ldots, b_{g} \mid\left[a_{1}, b_{1}\right] \ldots\left[a_{g}, b_{g}\right]=1\right\rangle
$$

с $2 g$ порождаюшими всякая подгруппа с меньшим числом порождаюших (не обязательно из множества $\left\{a_{1}, \ldots, a_{g}, b_{1}, \ldots, b_{g}\right\}$ ) свободна. В связи с этим второй автор ставил задачу нахождения условий на определяющие соотношения, при которых все подгрупшы конечно определенной группы с "малым" числом порождающих свободны. Такие условия были найдены в работе В. С. Губы [1], рассматривавшего подгрупшы с двумя порождающими. Кроме условия малого сокращения налагалось условие на "длинные 2-подслова" определяющих слов.

Для изучения групп со свободными $k$-порожденньми подгруппами при $k>2$ требовалось найти соответствующее обобщение последнего условия, что представлялось совсем не очевидным. Поэтому в [1] (а затем в $[2,11.75])$ поднимался вопрос об “общности" класса $m$-порожденных групп, в которых любая $(m-1)$-порожденная подгруппа свободна. Эта задача решена в настояшей заметке.

При фиксированных числах $m$ и $n$ порождающих и определяющих соотношений соответственно мы называем подкласс $\mathrm{C}$ конечных копредставлений обшим, если почти каждая группа с $m$ порождающими и $n$ соотношениями попадает в $\mathrm{C}$, если только определяюшие соотношения достаточно длинны. Нахождение общих свойств полезно хотя бы потому, что сочетание любого конечного числа общих свойств является опять-таки общим свойством групп.

Работа второго автора вьполнена при поддержке Международного научного фонда, грант MID000, и Российского фонда фундаментальных исследований, грант № 95-0101541. 
Для уточнения формулировок рассмотрим групповой алфавит $X_{m}=$ $\left\{x_{1}^{ \pm 1}, \ldots, x_{m}^{ \pm 1}\right\}$, где $m>1$, и множество циклически приведенных слов $\left\{r_{1}, \ldots, r_{n}\right\}$ в этом алфавите, имеюших длины $\left|r_{i}\right| \leqslant t$. Обозначим $N_{m, n, t}$ число всех копредставлений групा вида

$$
G=\left\langle x_{1}, \ldots, x_{m} \mid r_{1}=1, \ldots, r_{n}=1\right\rangle
$$

с этим условием, а $N_{m, n, t}^{f}$ - число таких копредставлений, что любая $(m-1)$-порожденная подгруппа в $G$ свободна (а, кроме того, $G$ действительно содержит свободные подгрупы любых конечных рангов).

Tеорема 1. Найдется число $\mathrm{c}=\mathrm{c}(m, n)>0$ такое, что отношение $N_{m, n, t}^{f} / N_{m, n, t}$ больше $1-\exp (-c t)$.

Как следствие получается ответ на вопросы из [1] и $[2,11.75]$. Именно, обозначим $N_{d}=N_{d}(m, n)$ число всех копредставлений груп вида (1) таких, что $\left|r_{1}\right|+\cdots+\left|r_{n}\right|=d$, где $r_{i}$ - циклически приведенное слово в алфавите $X_{m}$, а $N_{d}^{f}$ - число таких копредставлений среди них, что любая $(m-1)$-порожденная подгруппа в $G$ свободна.

ТЕОРема 2. При любом $m>1$ и любом фиксированном $n$

$$
\lim _{d \rightarrow \infty} \frac{N_{d}^{f}}{N_{d}}=1 .
$$

При решении основного вопроса вспомогательньг инструментом служат размеченные графы. Очевидно, например, что любое слово от переменных $x_{1}^{ \pm 1}, x_{2}^{ \pm 1}, x_{3}^{ \pm 1}$ может быть прочитано при обходе букета из трех циклов длины 1 , изображенном на рис. 1 .

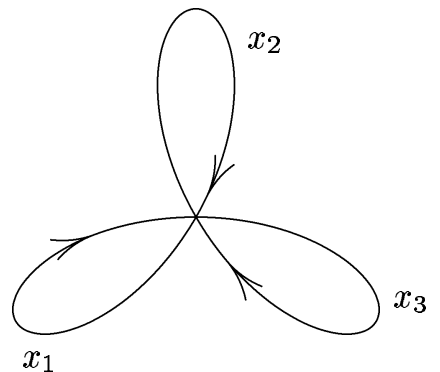

Рис. 1.

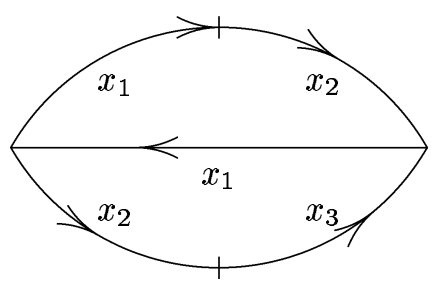

Рис. 2.

Если же рассмотреть граф рис. 2, то, обходя его, можно прочесть сколь угодно длинные слова, например:

$$
x_{1} x_{2} x_{1} x_{2} x_{3} x_{2}^{-1} x_{1}^{-1} x_{2} x_{3} x_{1} \ldots,
$$

и число таких слов экспоненциально растет вместе с их длиной, но в то же время экспоненциально убывает с ростом $\ell$ их доля среди всех несократимых слов длины $\ell$ от трех переменных. (Слово $x_{3}^{2}$ или $x_{1} x_{2} x_{1}^{-1}$ уже не читаемо по этому графу.) 
1. Размеченные графы и подгруппы. Пусть $\Gamma$ - связньй размеченньй граф, т.е. конечньй связный 1-комплекс, каждому ориентированному ребру $е$ которого приписана метка $\varphi(e)=x$, где $x \in X_{m}$, причем $\varphi\left(e^{-1}\right)=\varphi(e)^{-1}$. Одну из вершин $-o-$ считаем выделенной в $\Gamma$. Очевидно, что все слова, которые могут быть прочитаны на циклах графа $\Gamma$ с началом (и концом) в вершине $o$, образуют подгруппу $H=H(\Gamma)$ свободной группы $F_{m}=F\left(X_{m}\right)$ с базисом $X_{m}$ (хотя один и тот же элемент из $H$ может быть представлен разньми циклами из-за возможных сокращений).

Понятно, что $H(\Gamma)$ - гомоморфный образ фундаментальной групшы $\pi_{1}(\Gamma)$ при гомоморфизме, индуцированном размечающей функцией $\varphi$. Ясно также, что всякая конечно порожденная подгруппа $H \subset F_{m}$ может быть представлена таким образом. Достаточно выбрать в $H$ какую-то систему порождаюших $\left\{w_{1}, w_{2}, \ldots\right\}$ и отождествить начальные вершины простых циклов с метками $w_{1}, w_{2}, \ldots$ каждьй. (Под меткой $\varphi(p)$ симплициального пути $p=e_{1} \ldots e_{n}$ понимается слово $\left.\varphi\left(e_{1}\right) \ldots \varphi\left(e_{n}\right).\right) \mathrm{O}$ пользе такого задания подгрупшы свободной группы см., например, [3].

Если $G-m$-порожденная группа, заданная копредставлением (1), то всякая ее подгруппа $H$ также может быть задана некоторым графом $\Gamma,-$ достаточно рассмотреть какой-то ее прообраз при естественном гомоморфизме $F_{m} \rightarrow G$.

Размеченный связньй граф назовем приведенным, если в Г нет вершины, из которой выходит два ребра с одинаковыми метками, и нет вершин валентности 1 , кроме, может быть, отмеченной вершины $o$. Любой граф $\Gamma$, задающий подгрупу $H$, можно заменить приведенньм (см., например, [3]) с помощью преобразований 1-го рода (склеивание пары ребер с общим началом и одинаковьми метками) и 2-го рода (удаление вершины степени 1 вместе с входящим в нее ребром).

Под дугой будем понимать далее такой путь $p$, все вершины которого, кроме, быть может, начальной $p_{-}$и конечной $p_{+}$, отличны от выделенной вершины о и имеют валентность 2.

Для графа $\Gamma$, задающего подгруппу $H$ в группе $G$ с копредставлением (1), определим еще преобразование 3 -го рода:

Присоединение дуги. Пусть вершины $o_{1}$ и $o_{2}$ в $Г$ соединены некоторьм путем $p$ таким, что $\varphi(p) \equiv w$, и слово $w$ равно в $G$ некоторому слову $v$. Присоединим тогда к $\Gamma$ новый граф, состоящий из одной дуги $q$ с меткой $\varphi(q) \equiv v$ и имеюший с $\Gamma$ только $o_{1}=q_{-}$и $o_{2}=q_{+}($начало и конец пути $q)$ в качестве общих точек.

Обратное преобразование. Пусть $q$ - некоторая дуга в $\Gamma, q_{-}=o_{1}$ и $q_{+}=o_{2}$, причем вершины $o_{1}$ и $o_{2}$ можно соединить в $\Gamma$ другим путем $p$, таким, что $p$ и $q$ не имеют общих неориентированных ребер, а $\varphi(p)=\varphi(q)$ в группе $G$. Тогда разрешается удалить из $\Gamma$ все ребра и вершины пути $q$, кроме $o_{1}$ и $o_{2}$.

ЛЕмма 1. Если граф $\Gamma^{\prime}$ получен из графа Г с помощью нескольких 
преобразований 1-3-го рода (или обратных), то он определяет в $G$ my ж⿻е nодгрynny, что и граф $Г$.

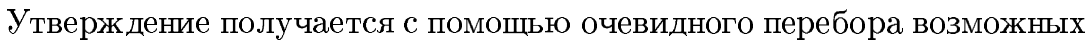
типов преобразований.

2. $\mu$-читаемые слова. Пусть $m \geqslant 2$. Назовем непустое несократимое слово $w$ длины $\ell$ в алфавите $X_{m}=\left\{x_{1}^{ \pm 1}, \ldots, x_{m}^{ \pm 1}\right\} \mu$-иитаемым $($ или $(\mu, m)$-читаемым) для некоторого $\mu$, где $0<\mu \leqslant 1$, если существует $X_{m}$-размеченный приведенньй граф $\Gamma$ со следующими свойствами:

(a) число ребер этого графа не больше $\mu \ell$;

(b) эйлерова характеристика графа $\Gamma$ больше $1-m$ (другими словами, $\pi_{1}(\Gamma)$ имеет ранг меньше $m$, т.е. в $Г$ меньше $m$ независимых циклов);

(c) слово $w$ может быть прочитано на некотором пути графа $\Gamma$.

Без второго условия определение становится бессодержательным, так как любое слово от $m$ переменных может быть прочитано на букете из $m$ окружностей длины 1 .

Оценим число $\mu$-читаемых слов длины $\ell$. Рассмотрим граф из определения $\mu$-читаемого слова. Поскольку в нем не более одной вершины валентности 1 , а эйлерова характеристика больше $1-m$, число вершин валентности $\geqslant 3$ не больше $2 m-3$. Из тех же соображений число максимальных дуг в $\Gamma$ не больше $3 m-4$. Отсюда понятно, что число топологических типов графов ограничено некоторой константой $\mathrm{C}(m)$.

Число возможных разбиений простых дуг графа определенного типа на $\leqslant \mu \ell$ ребер можно оценить сверху величиной

$$
\sum_{1 \leqslant k \leqslant[\mu \ell]}\left(\begin{array}{c}
3 m+k-5 \\
k-1
\end{array}\right) \leqslant(\mu \ell)^{3(m-1)} .
$$

Каждому из $\leqslant \mu \ell$ ребер приписывается одна из $2 m$ букв, что можно сделать не более чем $(2 m)^{\mu \ell}$ способами.

В итоге число размеченных графов из определения $\mu$-читаемого слова меньше

$$
\mathrm{C}(m)(\mu \ell)^{3(m-1)}(2 m)^{\mu \ell} .
$$

Оценим далее число несократимых слов длины $\ell$, которые читаются на данном графе $\Gamma$, начиная с произвольной вершины. Начальное ребро может быть выбрано $\leqslant 2 \mu \ell$ способами. После прохождения каждого ребра имеется $\leqslant 2 m-2$ способов для продолжения пути, поскольку валентность каждой вершины не больше $2 m-1$ (следует из ограничения на эйлерову характеристику и наличия не более одной вершины валентности 1). Значит, на $Г$ можно прочитать не более $2 \mu \ell(2 m-2)^{\ell-1}$ слов длины $\ell$. 
Вместе с (2) мы можем заключить, что число $\mu$-читаемых слов длины $\ell$ не больше

$$
2 C(m)(\mu \ell)^{3 m-2}(2 m)^{\mu \ell}(2 m-2)^{\ell-1} .
$$

Пусть теперь

$$
\mu<\log _{2 m}\left(1+\frac{1}{4(m-1)}\right) .
$$

Тогда из (3) вытекает следующее утверждение.

ЛЕмма 2. При условии (4) моэкно указать константу $k=k(\mu, m)$ такую, что число н-читаемых слов длины $\ell$ в алфавите $X_{m}$ меньwe $k(2 m-3 / 2)^{\ell}$.

3. Условие на определяющие слова. Рассмотрим в этом пункте $(\lambda, \mu)$-условие на систему определяющих слов. При этом выберем $\lambda>0$, а положительное $\mu$ - с условием (4).

Напомним (см. также [4]), что условие малого сокращения $\mathrm{C}^{\prime}(\lambda)$ на копредставление (1) означает, что если $R$ - множество всех циклических перестановок слов $\left\{r_{1}, \ldots, r_{n}\right\}$ и им обратных, то для различных $r, r^{\prime} \in R$ длина общего начала слов $r$ и $r^{\prime}$ меньше $\lambda \min \left(|r|,\left|r^{\prime}\right|\right)$.

ЛЕмма 3. Среди всех кортежсей $\left\{r_{1}, \ldots, r_{n}\right\}$ ииклически несократимых слов, длины которых не больие $t$, доля тех, которые удовлетворяют условию $\mathrm{C}^{\prime}(\lambda)$, не меньше чем $1-a(2 m-1)^{-\lambda t / 5}$ при $t \geqslant a$, әде $a=a(m, n, \lambda)>0$.

ДокАЗАтЕЛЬство. Очевидно, что число $N_{t, m}$ циклически несократимых слов длины $\leqslant t$ не меньше $(2 m-1)^{t-1}$, a, значит, число кортежей $N_{t, m, n}$, составленных из них, не меньше

$$
(2 m-1)^{n(t-1)} .
$$

Рассмотрим теперь циклически приведенное слово $r$ такое, что $t / 2<$ $|r| \leqslant t$, и допустим, что система $\{r\}$ не удовлетворяет условию $\mathrm{C}^{\prime}(\lambda)$. Легко видеть тогда, что в циклическом слове $r$ найдутся два непересекающихся подслова $u$ и $v$ таких, что $u=v^{ \pm 1}$ и $|u| \geqslant \lambda t / 4$. Но число всех слов с этим ограничением меньше $3 t^{2}(2 m-1)^{t-\lambda t / 4}$. Отсюда число кортежей $\left\{r_{1}, \ldots, r_{n}\right\}$ из слов длины $\leqslant t$, содержаших такое слово $r$, меньше $3 n t^{2}(2 m-1)^{t n-\lambda t / 4}$. Аналогично можно оценить сверху число кортежей $\left\{r_{1}, \ldots, r_{n}\right\}$, составленных из циклически приведенных слов с длинами в промежутке $(t / 2, t]$, таких, что для некоторых $i \neq j$ слова $r_{i}$ и $r_{j}$ имеют общее подслово длины $\geqslant \lambda t / 2$. Достаточно взять оценку $3 t^{2}(2 m-1)^{t n-\lambda t / 2}$. Наконец, число слов длины $\leqslant t / 2$ не больше $2 m(2 m-1)^{t / 2}$. Значит, число кортежей длины $n$, содержащих такие слова, меньше $2 m n(2 m-1)^{t(n-1)+t / 2}$. Складывая полученные оценки и относя сумму к $N_{t, m, n}$ (см. [5]), получаем утверждение леммы. 
ОПРЕДЕЛЕНИЕ. Под $(\lambda, \mu)$-условием для кортежа $\left\{r_{1}, \ldots, r_{n}\right\}$ циклически несократимых слов от $m$ переменных будем понимать справедливость следующих ограничений:

(а) кортеж $\left\{r_{1}, \ldots, r_{n}\right\}$ удовлетворяет условию $\mathrm{C}^{\prime}(\lambda)$;

(б) среди слов $r_{1}, \ldots, r_{n}$ нет истинных степеней;

(в) если $w$ является циклическим подсловом некоторого $r_{i}, 1 \leqslant i \leqslant n$, и $|w| \geqslant\left|r_{i}\right| / 2$, то $w$ не является $\mu$-читаемым.

Число циклически несократимых слов $r_{i}$ длины $\leqslant t$, имеющих вид $s^{d}$, не больше $(2 m-1)^{t / d} \leqslant(2 m-1)^{t / 2}$, если $d \geqslant 2$. Суммируя по всем $d=2, \ldots, t, i=1, \ldots, n$ и имея в виду оценку (5), получаем, что число всех кортежей $\left\{r_{1}, \ldots, r_{n}\right\}$ циклически несократимых слов длины $\leqslant t$, не удовлетворяющих условию (б), меньше

$$
n t(2 m-1)^{(n-1 / 2) t} .
$$

ЛЕмма 4. Если н удовлетворяет неравенству (4), то доля кортежей $\left\{r_{1}, \ldots, r_{n}\right\}$ чиклически приведенных слов в алфавите $X_{m}$ $c(\lambda, \mu)$-условием среди всех кортежей слов длины $\leqslant t$ не меньше $1-\exp (-\alpha t)$ при всех $t>T=T(\mu, \lambda, m, n)$ для некоторого $\alpha=\alpha(\mu, \lambda, m, n)>0$.

ДОКАЗАТЕЛЬСТВО. Оценим число кортежей, для которых нарушен пункт (в) $(\lambda, \mu)$-условия для $i=1$. Число таких слов $r_{1}$ по лемме 2 меньше

$$
\sum_{1 \leqslant d \leqslant t} d \sum_{d / 2 \leqslant \ell \leqslant d} k\left(2 m-\frac{3}{2}\right)^{\ell}(2 m-1)^{d-\ell}<2 k t^{2}\left(2 m-\frac{5}{4}\right)^{t} .
$$

Значит, число кортежей $\left\{r_{1}, \ldots, r_{n}\right\}$, где $r_{1}$ не удовлетворяет пункту (в), меньше $2 k t^{2}(2 m-5 / 4)^{t}(2 m-1)^{t(n-1)}$. Отсюда, а также из утверждения леммы 3 и оценок (5), (6), следует утверждение леммы.

4. Подгруппы с малым числом порождающих. Ввиду леммы 4, при доказательстве теорем достаточно доказать свободу подгрупп с $\leqslant(m-1)$ порождаюшими в любой группе $G$ c $(\lambda, \mu)$-условием для некоторых $\lambda$ и $\mu$ с условием (4) соответственно. (При $t \leqslant T$ достаточно выбрать просто положительный параметр $\mathrm{c}=\mathrm{c}(m, n)$ достаточно малым, имея в виду, что $N_{m, n, t}^{f} \geqslant 1$, можно взять пустые слова $r_{1}, \ldots, r_{n}$ в (1).) Пусть теперь

$$
\lambda \leqslant \frac{\mu}{15 m+3 \mu}<\frac{1}{6} .
$$

Доказывая "от противного", рассмотрим в $G$ подгруппу $H$, минимальное число порождаюших $s$ которой $\leqslant m-1$. В этом случае размеченньй граф $\Gamma$, задающий подгруппу $H$, имеет эйлерову характеристику $>1-m$. 
Будем считать, что граф Г выбран с минимальным числом ребер. В частности, он является приведенньм.

Предполагая, что $H$ не свободна, имеем нетривиальньй элемент в ядре естественного гомоморфизма $\pi_{1}(\Gamma) \rightarrow H$, т.е. в $\Gamma$ есть цикл $p$, метка которого - тривиальное в $G$ слово. При этом путь $p$ редуцирован, т.е. не содержит подпутей вида $e e^{-1}$. В силу приведенности графа $\Gamma$ слово $w \equiv \varphi(p)$ также приведено.

По лемме Гриндлингера для $\mathrm{C}^{\prime}(\lambda)$-групп при $\lambda \leqslant 1 / 6$ (см. [4]), в $w$ найдется подслово $v$, которое одновременно является подсловом циклической перестановки $r$ некоторого определяющего слова $r_{i}^{ \pm 1}$, причем $|v|>(1-3 \lambda)\left|r_{i}\right|$.

Пусть $p=p_{1} \ldots p_{t}$ - разложение пути $p$ на максимальные дуги, а слово $v$ читается на дугах $\bar{p}_{k}, \bar{p}_{k+1}, \ldots, \bar{p}_{\ell}$, т.е. $v \equiv v_{k} v_{k+1} \ldots v_{\ell}$, где $\varphi\left(\bar{p}_{k+1}\right) \equiv$ $v_{k+1}, \ldots, \varphi\left(\bar{p}_{\ell-1}\right) \equiv v_{\ell-1}$, a $\varphi\left(\bar{p}_{k}\right) \equiv v_{k}, \varphi\left(\bar{p}_{\ell}\right) \equiv v_{\ell}$, где $\bar{p}_{k}-$ конец дуги $p_{k}, \bar{p}_{\ell}-$ начало дуги $p_{\ell}, \bar{p}_{j}=p_{j}$ при $j=k+1, \ldots, \ell-1$.

Рассмотрим несколько возможностей.

1. Среди слов $v_{k}, v_{k+1}, \ldots, v_{\ell}$ найдется слово $v_{j}$, где $\left|v_{j}\right| \geqslant 5 \lambda|r|$.

В силу условий (а) и (б), дуга $p_{j}^{ \pm 1}$ не может тогда дважды встречаться в ряду $p_{k+1}, \ldots, p_{\ell-1}$. Эти же условия означают, что если $j \neq k, \ell$ и $p_{j}^{ \pm 1}$ дважды встречается в ряду $p_{k}, \ldots, p_{\ell}$, скажем $p_{j_{0}}=p_{j}$ при $j_{0} \neq j$, то $j_{0}=k$ (или $j_{0}=\ell$ ), причем в этом случае $\left|v_{k}\right|<\lambda|r|$ (соответственно $\left.\left|v_{\ell}\right|<\lambda|r|\right)$.

Если $p_{j}^{ \pm 1}$ встречается только один раз среди $p_{k}, \ldots, p_{\ell}$, то можно провести преобразование 3-го рода, соединив сначала дугой длины $|r|-|v|$ с меткой $u$ такой, что $r \equiv v u^{-1}$, вершины $\left(\bar{p}_{k}\right)_{-}$и $\left(\bar{p}_{\ell}\right)_{+}$, а затем выбросив дугу $p_{j}$ из $\Gamma$ (мы пользуемся тем, что $\varphi(u)=\varphi(v)$ в $G$ ). При этом число ребер в $Г$ уменьшится, ибо $|r|-|v|<3 \lambda|r|<\left|p_{j}\right|$, а эйлерова характеристика не изменится. Получаем противоречие с минимальностью в выборе $\Gamma$.

Во втором варианте дугой соединим вершины $\left(\bar{p}_{k}\right)_{+}$и $\left(\bar{p}_{\ell}\right)_{+}$, если $\left|v_{k}\right|<\lambda|r|$. Ее метка будет иметь длину $|r|-|v|+\left|v_{k}\right|<4 \lambda|r|$ (или $|r|-|v|+\left|v_{k}\right|+\left|v_{\ell}\right|<5 \lambda|r|$, если еше и $\left.\left|v_{\ell}\right|<\lambda|r|\right)$, и она равна в $G$ элементу, представленному словом $v_{k+1} \ldots v_{\ell}$. Поэтому, выбрасывая затем дугу $p_{j}$, получаем противоречие, как и вьше.

Рассмотрим еще возможность $p_{k}=p_{\ell}^{ \pm 1}$ и $\left|v_{k}\right| \geqslant 5 \lambda|r|$. При $p_{k}=p_{\ell}^{-1}$, как и вьше имеем, что $\left|v_{\ell}\right|<\lambda|r|$ из условия $\mathrm{C}^{\prime}(\lambda)$. Если же $p_{k}=p_{\ell}$, то при разложении $p_{k}=q^{\prime} q$, где $|q|=\left|v_{k}\right|-[\lambda|r|]$, ребра дуги $q$ уже не встречаются в подпути пути $p_{\ell}$, на котором написано слово $v_{\ell}$. В этом случае противоречие получается при стирании дуги $q$ (и добавлении новой дуги длины $<4 \lambda|r|)$.

2. $\left|v_{j}\right|<5 \lambda|r|$ для $j=k, \ldots, \ell$.

В этом случае заметим предварительно, что, ввиду приведенности графа Г и ограничения на эйлерову характеристику, число различных максимальных дуг в $\Gamma$ не превосходит $3 m-1$. Поэтому слово $v$ прочитывает- 
ся на некотором связном подграфе графа $\Gamma$ с эйлеровой характеристикой $\geqslant 1-m$ и числом ребер $\leqslant 3 m 5 \lambda|r| \leqslant \mu(1-3 \lambda)|r|<\mu|v|$ по выбору $\mu$ и $\lambda$. Поскольку $v$ - подслово в $r$ длины $>|r| / 2$, получили противоречие с $(\lambda, \mu)$-условием.

Для окончания доказательства достаточно найти в любой группе рассматриваемого класса свободную подгруппу ранга 2 . При этом можно дополнительно считать, что $\left|r_{i}\right| \geqslant 12$ для $i=1, \ldots, n$. (Это условие нарушено для кортежей, число которых экспоненциально мало по сравнению с $N_{m, n, t}$, так как оно меньше $n(2 m-1)^{(n-1) t+11}$.)

Выберем число $d$ так, что $d=\max _{1 \leqslant i \leqslant n}\left|r_{i}\right|$, и проверим, что $x_{1}^{d}$ и $x_{2}^{d}$ порождают свободную подгрупу. Допустив, что $w\left(x_{1}^{d}, x_{2}^{d}\right)=1$ в $G$ для непустого циклически несократимого слова $w$, получаем с помощью цитированной вьше леммы Гриндлингера, что в некотором слове $r_{i}^{ \pm 1}$ есть циклическое подслово $v \equiv x_{j}^{\ell}$, где $j=1$ или $j=2$, а $\ell>\left|r_{i}\right| / 4$. Но в случае $\left|r_{i}\right| \geqslant 12$ это противоречит условиям (а), (б) и (7), поскольку два различных циклических вхождения слова $x_{j}^{\ell-1}$ в $r_{i}^{ \pm 1}$ имеют длину $\geqslant\left|r_{i}\right| / 6$.

Легко видеть, что при доказательстве теоремы 2 можно пренебречь числом кортежей $\left\{r_{1}, \ldots, r_{n}\right\}$ таких, что $\left|r_{1}\right|+\cdots+\left|r_{n}\right|=d$, но $\left|r_{i}\right|<\sqrt{d}$ хотя бы для одного $i \leqslant n$. Предполагая же, что $\left|r_{i}\right|=d_{i} \geqslant \sqrt{d}$ для всех $i$, получим для всех отношений $N_{d_{1} \ldots d_{n}}^{f} / N_{d_{1} \ldots d_{n}}$ равномерно близкие к 1 (т.е. независимые от выбора чисел $d_{1} \ldots d_{n}$ таких, что $d_{1}+\cdots+d_{n}=d$ ) оценки таким же образом, как и в леммах 3 и 4 вьше. Для завершения доказательства теоремы 2 параметры $\mu$ и $\lambda$ можно выбрать так же, как и в теореме 1.

В последующей статье предполагается снять ограничение " $<m$ " на число порождающих, заменив произвольной наперед заданной гранищей " $<L$ " (где возможно $L \gg m$ ), доказав общность свойства свободы всех $\leqslant L$-порожденных подгрупп группы $G$ бесконечного индекса.

Московский государственный университет

Поступило

им. М.В. Ломоносова

05.01 .95

E-mail : olsh@nw.math.msu.su

\section{СПИСОК ЦИТИРОВАННОЙ ЛИТЕРАТУРЫ}

[1] Губа В. С. Об условиях, при которых 2-порожденные подгруппы в группах с малым сокращением свободны // Изв. вузов. Матем. 1986. № 7. С. 12-19.

[2] Коуровская тетрадь. (Нерешенные вопросы теории групп). Новосибирск: Наука, 1990. 11-е изд.

[3] Margolis S. W., Meakin J. C. Free inverse monoids and graph immersions // Int. J. Algebra and Comput. 1993. V. 3. №1. P. 79-100.

[4] Линдон Р., Шупп П. Комбинаторная теория групп. М: Мир, 1980. 